\title{
Métodos para o Desenvolvimento de Jogos Digitais de Lógica: Uma Revisão Sistemática
}

\author{
Lucy Mari Tabuti, Ricardo Nakamura \\ Escola Politécnica - Universidade de São Paulo (USP) \\ Av. Prof. Luciano Gualberto, travessa 3, 380 - CEP 05508-010 - Butantã - São Paulo - \\ SP - Brasil \\ lucymari@gmail.com,ricardo.nakamulra@poli.ups.br
}

\begin{abstract}
With the fast development of technology and its easy access to a large number of users - especially mobile devices - digital games are becoming more popular and are used more often, including academic environment use. Although, for the digital games - especially logic games - to have a qualitative result in education they must be developed with criteria and methodology that assure learning. This article presents a systematic review that aims to identify and understand the current methodologies for the development of digital logic games qualified for education. The review unveils that studies which use or define methodologies for the development of digital logic games have not been yet explored in literature.
\end{abstract}

Resumo. Com o avanço da tecnologia e a crescente facilidade de sua utilização, principalmente, em dispositivos móveis, os jogos digitais têm se popularizado, inclusive nos meios acadêmicos. No entanto, para que os jogos digitais, particularmente, os de lógica, tenham um alcance qualitativo na educação, é necessário que sejam desenvolvidos com critérios de metodologias que garantam o aprendizado. Assim, este artigo apresenta uma revisão sistemática com o propósito de identificar e estudar os métodos existentes para o desenvolvimento de jogos digitais de lógica qualificados para a educação. A revisão mostrou que estudos que utilizam ou definem métodos deste tipo ainda não foram explorados na literatura.

\section{Introdução}

Com o desenvolvimento da tecnologia de Informação, crianças e adolescentes têm à disposição uma abundância de dispositivos móveis com diferentes aplicativos que lhes detêm a atenção. É sabido também que a utilização dos jogos de lógica físicos é importante para o desenvolvimento lógico e cognitivo da criança e do adolescente, independentemente da área de conhecimento em que um dia ingressarão.

Os jogos de lógica são os mais antigos e têm por objetivo desafiar o jogador, pois apresentam problemas que necessitam de raciocínio e paciência. Cuperschmid e Hildebrand (2013) dizem que quando os jogos de lógica são apresentados por um meio digital, eles podem ter equivalentes no mundo real como, por exemplo, os jogos de cartas ou jogos de tabuleiro, ou não ter qualquer relação com o mundo real como, por exemplo, o jogo Tetris. Para Cuperschmid e Hildebrand (2013), os jogos de lógica, além 
de serem divertidos de resolver, desafiam mais a mente do que desafiam reflexos. Isso porque desafiam intelectualmente para a resolução de problemas, não só o pensamento, mas também a paciência.

Fernandes (2010) disse que, como jogos de lógica, "os tradicionais jogos de tabuleiro, xadrez, quebra-cabeça, batalha naval, entre outros, são os mais semelhantes aos utilizados como paradidáticos nas escolas" e podem ser trabalhados tanto analogicamente como digitalmente.

Para Ramos (2013), o desenvolvimento de um trabalho pedagógico, que utilize jogos de lógica ou jogos em geral, ajuda no exercício e no desenvolvimento de aspectos cognitivos do estudante de forma a torná-los mais lúdicos e prazerosos. Além disso, é sabido que o jogo beneficia os aspectos sociais, afetivos e cognitivos do estudante permitindo desenvolver outras características como a imaginação, a imitação e a regra.

Para os jogos que desafiam a lógica, Ramos (2013) direcionou-se aos "problemas que mobilizam o jogador a pensar, levantar hipóteses, experimentar, planejar, testar, realizar cálculos", contribuindo para "o desenvolvimento do raciocínio lógico, do planejamento, da percepção visual e da atenção". Além disso, há aqueles jogos de lógica que exigem o desenvolvimento de uma estratégia e do raciocínio lógico para resolver o desafio lógico ou mesmo para vencer o adversário.

Segundo Lee e Jones apud Ramos (2013), "a educação que tem como objetivo o desenvolvimento do cérebro, envolve a aprendizagem de exercícios e práticas destinadas a melhorar e transformar a maneira como o cérebro funciona". Assim, os jogos de lógica exercem papel fundamental e com grande potencial em contribuir com $\mathrm{o}$ desenvolvimento da aprendizagem e da educação de forma integral.

Os atuais alunos de ensino fundamental e médio são alunos cujas gerações já nasceram com a internet. Segundo Fraiman (2013), "esses jovens se desenvolveram com um modelo mental diferente, as sinapses aconteceram de forma diferente em relação às gerações analógicas, nascidas antes da internet".

Segundo Freire (2011), "Ninguém ignora tudo. Ninguém sabe tudo. Todos nós sabemos alguma coisa. Todos nós ignoramos alguma coisa. Por isso, aprendemos sempre". Porém, o aprendizado desta nova geração de estudantes precisa estar conectado ao mundo digital, por ser uma geração que nasceu com a internet.

Por este motivo, é importante a busca de uma educação ou modelo pedagógico voltado para os estudantes dessas gerações atuais, proporcionando uma educação mais motivadora e autodidata. Para Fraiman (2013), "o método de transmissão de conhecimento presente em nossas escolas precisa ser modificado rapidamente para uma versão digital, (...), pois só assim, conseguiremos estabelecer um protocolo de comunicação eficiente com elas para atingirmos o sucesso no aprendizado".

Ott e Pozzi (2011) afirmam, porém, que há uma necessidade de aprofundar os estudos para o desenvolvimento desses jogos de lógica digital, de forma a garantir que, quando utilizados com o propósito educacional, capacitem a criatividade das crianças e adolescentes.

Mäyrä, Holopainen, e Jakobsson (2012) apresentam os campos em que as pesquisas com os jogos digitais têm evoluído por meio de alguns artigos apresentados 
no simpósio de Simulation \& Gaming: An Interdisciplinary Journal. Essas pesquisas podem ser utilizadas para verificar, por exemplo, o movimento a partir dos jogos clássicos de computadores em direção aos jogos nas formas híbridas; os jogos em dispositivos móveis; os jogos online; e a inovação das interfaces sendo adotadas nas propostas desses jogos. Além disso, a análise de métodos úteis que auxiliam na evolução dos estudos de jogos como um campo próprio.

Por isso, surgiu a ideia do estudo das metodologias existentes para a digitalização dos jogos de lógica do meio físico para o meio virtual. Os jogos de lógica digitais são ferramentas virtuais que podem ser utilizadas no processo de ensinoaprendizagem, otimizando o trabalho e o tempo de professores e estudantes da geração atual (Fraiman, 2013 e Sato, 2009).

A metodologia para o desenvolvimento dos jogos de lógica digitais precisa ter características cujas gerações atuais esperam: o prazer, o sentido no aprendizado, a competição, o enredo e a aventura (Fraiman, 2013 e Sato, 2009). Essas características são observadas nos diversos games de sucesso garantidos por essa nova geração de estudantes. Para Fraiman (2013), "a transmissão do conhecimento precisa estar na VIBE da nova geração (Vibração, Importância, Belo e Entretenimento)". A presença destas características nos métodos de desenvolvimento dos jogos de lógica digital tem o objetivo de tornar o aprendizado prazeroso, o que o torna mais significativo.

Além disso, como essa nova geração de estudantes está conectada o tempo todo com seus smartphones, os aplicativos digitais, como os propostos jogos de lógica digitais, podem ser facilmente utilizados por serem "focados, simples de usar, ágeis e muito mais conectados às novas gerações".

Este artigo propõe uma revisão sistemática para verificar as metodologias existentes para o desenvolvimento de jogos de lógica digitais e como se dá o processo de aprendizado e das competências, habilidades e estratégias, oferecidas por esses jogos.

Apesar de esta pesquisa envolver os jogos digitais de lógica de forma generalizada, é importante ressaltar que existem literaturas que realizam estudos dos métodos de desenvolvimentos de jogos digitais de aprendizagem específicos.

Chen et al. (2014) apresentou um método de desenvolvimento de um jogo digital para o ensino da matemática, em que a utilização do jogo pelos estudantes apresenta um melhor aproveitamento do aprendizado. Hsu e Shih (2012) apresentaram um método para desenvolvimento de jogos digitais considerando a interação com o usuário, esperando que o jogo digital possa ser utilizado como consulta ao aprendizado. Mohebzada e Bhojani (2011) apresentaram um método de desenvolvimento de um sistema de aprendizado baseado em jogos, que é utilizado pelos estudantes que criam células a partir de cubos. Dantas (2013) et al. apresentaram uma metodologia que utiliza os jogos digitais no desenvolvimento da habilidade para resolução de problemas.

\section{Metodologia de Revisão}

Segundo os critérios de Kitchenham (2004), o desenvolvimento desta revisão sistemática está dividido em três etapas: a de planejamento, a de condução e a da documentação, de forma que cada etapa pode ser dividida em alguns estágios para um melhor entendimento do processo da revisão sistemática. A etapa de planejamento, na 
qual os objetivos da revisão são definidos, refere-se à fase de identificação da necessidade de uma revisão e à fase do desenvolvimento de um protocolo de revisão de como será realizada a pesquisa e como os estudos serão validados.

A etapa de condução, na qual o protocolo é desenvolvido, refere-se à fase de identificação da pesquisa; à seleção de estudos primários com definição dos mecanismos para revisão sistemática; à avaliação da qualidade do estudo que determina quais estudos são eliminados a partir de um critério; à extração e monitoração dos dados que verifica os assuntos pertinentes dos estudos selecionados de acordo com o protocolo; e à síntese dos dados com a análise e a comparação a partir das informações extraídas.

A etapa de documentação, em que os dados sintetizados podem ser comparados com outras informações, refere-se ao desenvolvimento do relatório de revisão e à validação do relatório.

\subsection{Perguntas para a Pesquisa}

Uma vez que o objetivo desta pesquisa é identificar e analisar os métodos existentes para o desenvolvimento de jogos de lógica digitais, as perguntas para a pesquisa seguiram as ideias apresentadas por Biolchini et al. (2005) e Kitchenham (2009): Com qual intensidade ocorrem as pesquisas relacionadas às metodologias para 0 desenvolvimento de jogos de lógica digitais? (P1); Quais temas de pesquisa estão sendo abordados quando relacionadas aos métodos para o desenvolvimento de jogos de lógica digitais? (P2); Quais abordagens estão sendo consideradas pelos pesquisadores que têm pesquisado métodos para o desenvolvimento de jogos de lógica digitais? (P3); e Quais métodos empíricos de pesquisa estão sendo utilizados pelos pesquisadores das metodologias para o desenvolvimento de jogos de lógica digitais? (P4).

Para responder a P1, foi verificado o ano, país e continente de cada publicação e os resultados estão apresentados na forma gráfica. E, para responder as demais questões, foi necessário classificar cada uma das perguntas de acordo com critérios estabelecidos para esta pesquisa que abrange a abordagem e o método da pesquisa, os quais estão detalhados na Seção 3.

\subsubsection{Base de Dados}

Uma revisão sistemática deve identificar as fontes principais de dados sobre determinado tema. Os estudos relacionados devem estar publicados em revistas em diversas áreas e conferências sobre entretenimento computacional e jogos digitais.

As bases de pesquisa foram Association for Computing Machinery (ACM), IEEE Computer Society (IEEE), Springer e Elsevier e algumas conferências, tais como "Computer Games and Digital Entertainment", "Conference on Interactive Entertainment", "E-learning and games, edutainment technologies", "Advances in Computer-Human Interactions", "Digital Games and Intelligent Toys Based Education" e "Digital Game and Intelligent Toy Enhanced Learning", entre outras.

A pesquisa em cada uma dessas bibliotecas digitais teve por base as palavraschaves que foram definidas de acordo com as perguntas para a pesquisa: digital games, que deveria aparecer no título do artigo, logic que deveria aparecer no resumo do artigo, 
e variações de methodology, method, approach, technique, development e model que deveria aparecer no resumo do artigo. Esta pesquisa incluiu artigos nas línguas portuguesa e inglesa, porém, a maioria dos artigos encontrados foi no idioma inglês. A partir dos critérios estabelecidos, 346 artigos foram selecionados para o desenvolvimento desta revisão.

\subsection{Os Critérios de Inclusão e Exclusão}

Apesar de a pesquisa inicial ter sido realizada a partir das palavras-chave determinadas como relevantes para esta pesquisa, nem todos os artigos abordavam diretamente as perguntas relacionadas com o objetivo desta pesquisa. Desta forma, alguns critérios de inclusão e exclusão foram definidos: 1. Com base no título: o critério mais simples, no qual o título de cada artigo foi lido e julgado como importante ou não para a área de pesquisa; 2. Com base no resumo: se o critério com base no título não foi o suficiente, pois as informações eram vagas e incompletas, a leitura do resumo foi realizada para verificar a aderência do artigo à área de pesquisa; e 3. Com base no texto: se os critérios com base no título e no resumo não foram suficientes, pois as informações eram vagas e incompletas, a leitura de todo o artigo foi realizada para não deixar dúvidas quanto à importância do artigo à área de pesquisa.

Após aplicar o primeiro critério, 346 artigos foram selecionados. Após a aplicação do segundo critério, 70 artigos foram selecionados e, após o terceiro critério, apenas 31 artigos foram finalmente selecionados para o estudo desta revisão.

\subsection{Coleta e Análise dos Dados}

A partir dos artigos pesquisados e os selecionados pelos critérios de inclusão e exclusão, o próximo passo foi extrair as principais informações de cada artigo para responder às perguntas definidas na etapa de planejamento. As informações extraídas de cada artigo foram tipo de publicação; ano de publicação, país e continente; e classificação pelos critérios estabelecidos para esta pesquisa.

As informações tabuladas consideraram o número de artigos publicados por ano (P1); o número de artigos por país e continente (P1); e classificação dos estudos em critérios que envolvem tema, abordagens, métodos e relevância à pesquisa (P2 à P4).

\section{Resultados}

Nesta seção, os resultados da análise estatística do conjunto de artigos selecionados são apresentados de acordo com os critérios definidos. Para uma melhor apresentação das informações, os artigos foram classificados com as siglas de A1 até A31, conforme apresentados no Quadro 1.

Quadro 1. Artigos classificados para esta pesquisa

\begin{tabular}{|c|c|}
\hline A1 & $\begin{array}{c}\text { Ying, M., Yang, K. (2013) "A game-based learning system using the ARCS model and fuzzy logic". Journal of } \\
\text { Software. USA. }\end{array}$ \\
\hline A2 & $\begin{array}{c}\text { Arachehilage, N. A. G., Love, S. (2013) "A game design framework for avoiding phishing attacks". Computers in } \\
\text { Human Behavior, Volume 29, Issue 3, Pages 706-714. }\end{array}$ \\
\hline A3 & $\begin{array}{c}\text { Connolly, T.M., Boyle, E.A., MacArthur, E., Hainey, T., Boyle, J.M. (2012) "A systematic literature review of } \\
\text { empirical evidence on computer games and serious games". Computers \& Education, Volume 59, Issue 2, } \\
\text { Pages 661-686. }\end{array}$ \\
\hline A4 & $\begin{array}{l}\text { Viana, J.R.M., Viana, N.P., Trinta, F.A.M., Carvalho, W.V. (2014) "A systematic review on software engineering } \\
\text { in pervasive games development". Computer Games and Digital Entertainment (SBGAMES), 2014 Brazilian } \\
\text { Symposium on, pp.51,60. }\end{array}$ \\
\hline
\end{tabular}




\section{Quadro 1. Artigos classificados para esta pesquisa}

\begin{tabular}{|c|c|}
\hline A5 & $\begin{array}{l}\text { ang, P., Lau, R.W.H., Pan, Z., Wang, J. Song, H. (2014) "An eigen-based motion retrieval method for real-time } \\
\text { animation". Computers \& Graphics, Volume 38, Pages 255-267. }\end{array}$ \\
\hline A6 & $\begin{array}{l}\text { arner, J., Wood, G., Pijnappel, S. Murer, M., Mueller, F.F. (2013) "Combining moving bodies with digital } \\
\text { elements: design space between players and screens". In Proceedings of The 9th Australasian Conference on } \\
\text { Interactive Entertainment: Matters of Life and Death (IE '13). ACM, USA, Article 17, } 10 \text { pages. }\end{array}$ \\
\hline A7 & $\begin{array}{l}\text { Jogueira, P.A., Aguiar, R., Rodrigues, R., Oliveira, E. (2014) "Computational Models of Players' Physiological } \\
\text { based Emotional Reactions: A Digital Games Case Study". Web Intelligence (WI) and Intelligent Agent } \\
\text { Technologies (IAT), } 2014 \text { IEEE/WIC/ACM International Joint Conferences on, vol.3, pp.278,285. }\end{array}$ \\
\hline A8 & $\begin{array}{l}\text { Robertson, J., Howells, C. (2008) "Computer game design: Opportunities for successful learning". Comput. } \\
\text { Educ. 50, 2, 559-578. }\end{array}$ \\
\hline A9 & $\begin{array}{l}\text { chalk, A., Juan, J., Perez. J.P. (2005) "Concrete Data Structures as Games". Notes Theor. Comput. Sci. 122, 193- } \\
210 .\end{array}$ \\
\hline A10 & $\begin{array}{l}\text { est, J. (2006) "Conditional reasoning in the context of strategy development in the log } \\
\text { 'mastermind"'. North American Journal of Psychology on, vol. 8. USA. }\end{array}$ \\
\hline A11 & $\begin{array}{l}\text { ouceiro, R.M., Papastergiou, M., Kordaki, M., Veloso, A.I. (2013) "L } \\
\text { for the learning of Information and Communication Technologies } \\
\text { sport science students". Education and Information Technologies, } 5\end{array}$ \\
\hline A12 & $\begin{array}{l}\text { su, Q.C., Chou, J.H. (2012) "Development of 3D Virtual Multi-Pyramid a } \\
\text { and Middle Schools' Students". Computer, Consumer and Control (IS3C } \\
\text { vol., no., pp.132,136, 4-6. }\end{array}$ \\
\hline A13 & $\begin{array}{l}\text { Chung, I., Wu, Y. (2011) "Digital Educational Games in Science Learning: A Review of Empirical Research". } \\
\text { In Proceedings of the 6th international conference on E-learning and games, edutainment } \\
\text { technologies (Edutainment'11), Springer-Verlag, Berlin, Heidelberg, 512-516. }\end{array}$ \\
\hline A14 & $\begin{array}{l}\text { Irove, F., Bourgonjon, J., Looy, J.V. (2012) "Digital games in the classroom? A contextual } \\
\text { adoption intention of digital games in formal education". Computers in Human Behavior } \\
\text { Pages 2023-2033. }\end{array}$ \\
\hline 15 & $\begin{array}{l}\text { Iong, J., Hwang, M., Tam, K., Lai, Y., Liu, L. (2012) "Effects of cog } \\
\text { performance: A GridWare analysis". Comput. Hum. Behav.28, 3, 920-92 }\end{array}$ \\
\hline A16 & $\begin{array}{l}\text { oyle, E.A., Connolly, T.M., Hainey, T., Boyle, J.M. (2012) "Engagemen } \\
\text { systematic review". Comput. Hum. Behav. 28, 3, 771-780. }\end{array}$ \\
\hline 17 & $\begin{array}{l}\text { aw, E.L., Sun, X. (2012) "Evaluating user experience of adaptive digital educa } \\
\text { Theory". Int. J. Hum.-Comput. Stud. } 70,7,478-497 .\end{array}$ \\
\hline A18 & $\begin{array}{l}\text { rapolli, F., Brocco, A., Malatras, A., Hirsbrunner, B. (2010) "FLEXIBLE RULE } \\
\text { Game Development Framework". In Proceedings of the } 2010 \text { Third Internationa } \\
\text { Computer-Human Interactions (ACHI '10). IEEE Computer Society, Washington, }\end{array}$ \\
\hline 19 & $\begin{array}{l}\text { Neville, D.O., Shelton, B.E. (2010) "Literary and Historical 3D Digital Game-Based Learning: Design } \\
\text { Guidelines". In OAI, Simulation \& Gaming 01/2010. }\end{array}$ \\
\hline $\mathrm{A} 20$ & $\begin{array}{l}\text { ickmeier-Rust, M.D., Hockemeyer, C., Albert, D., Augustin, T. (2008) "Micro Ada } \\
\text { Knowledge Assessment in Educational Games". Digital Games and Intelligent Toys Ba } \\
\text { Second IEEE International Conference on, pp.135,137, 17-19. }\end{array}$ \\
\hline $\mathrm{A} 21$ & $\begin{array}{l}\text { onge, M., Stirling, C. (2002) "Model Checking Games for Branching Time Logics". J. Logic Computat., Vol. } 12 \\
\text { No. 4, pp. 623-639. }\end{array}$ \\
\hline 22 & $\begin{array}{l}\text { utierrez, J., Bradfield, J. (2009) "Model-checking games for fixpoint logics with par } \\
\text { International Conference, CONCUR 2009, Bologna, Italy, pp 354-368. }\end{array}$ \\
\hline & räDel, W. (2013) "Model-checking games for logics of imper \\
\hline $\mathrm{A} 24$ & $\begin{array}{l}\text { ester, S. (2014) "Model-checking Quantitative Alternating-time Temporal Logic o } \\
\text { arXiv: } 1409.5681 \text {. vol. 1. [cs.LO]. }\end{array}$ \\
\hline $\mathrm{A} 25$ & $\begin{array}{l}\text { acke, H. (2012) "Narrative rules? Story logic and the str } \\
\text { Vol. 27, No. 3. Published by Oxford University Press. }\end{array}$ \\
\hline A26 & $\begin{array}{l}\text { Chiu, W., Chiou, S. (2010) "On Design Methodology and Practice of Bionics". In Proceedings of the } 2010 \text { Third } \\
\text { IEEE International Conference on Digital Game and Intelligent Toy Enhanced Learning (DIGITEL '10). } \\
\text { IEEE Computer Society, Washington, DC, USA, 210-212. }\end{array}$ \\
\hline $\mathrm{A} 27$ & $\begin{array}{l}\text { Ravenscroft, A. (2007) "Promoting thinking and conceptual change with digital dialogue games". J. Comp. } \\
\text { Assisted Learning 01/2007; vol. 23, pp. 453-465. }\end{array}$ \\
\hline A28 & $\begin{array}{l}\text { Ampatzoglou, A., Stamelos, J. (2010) "Software engineering research for computer games: A systematic review". } \\
\text { Inf. Softw. Technol. 52, 9, 888-901. }\end{array}$ \\
\hline A29 & $\begin{array}{l}\text { Cruz, A., Coelho, A., Sousa, A. (2011) "Technical Analysis and Approaches for Gam } \\
\text { Life". Information Systems and Technologies (CISTI), } 2011 \text { 6th Iberian Conference } \\
\end{array}$ \\
\hline $\mathrm{A} 30$ & $\begin{array}{l}\text { Wu, B. (2013) "Theoretical Foundation for Lecture Games". NTNU-trykk. Doctoral Theses at NTNU, 1503-8181. } \\
\text { 2013: 195. }\end{array}$ \\
\hline & kt, M., Lenhardt, R., Worrell, J. (2011) "Two vari \\
\hline
\end{tabular}

Para responder à $\mathrm{P} 1$ sobre os estudos dos métodos de desenvolvimento de jogos digitais de lógica, pode-se observar, na Figura 1, que as pesquisas intensificaram-se a 
partir de 2010 e, observando a Figura 2, que o continente Europeu, em particular o Reino Unido, é o lugar onde as pesquisas ocorrem com mais frequência.

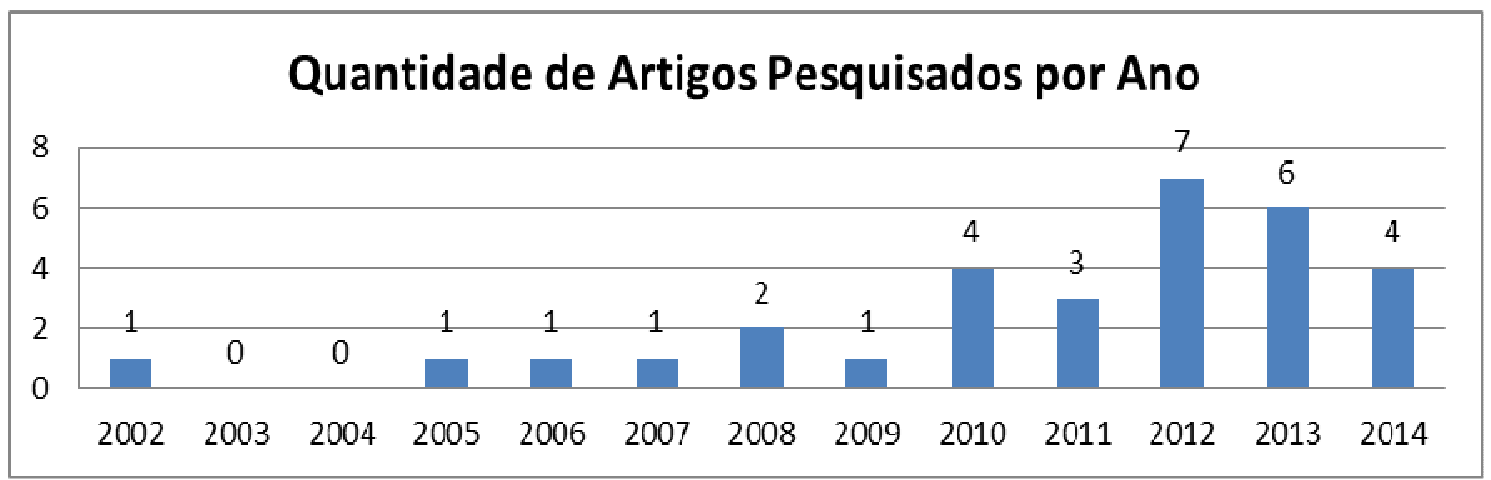

Figura 1. Quantidade de artigos pequisados por ano
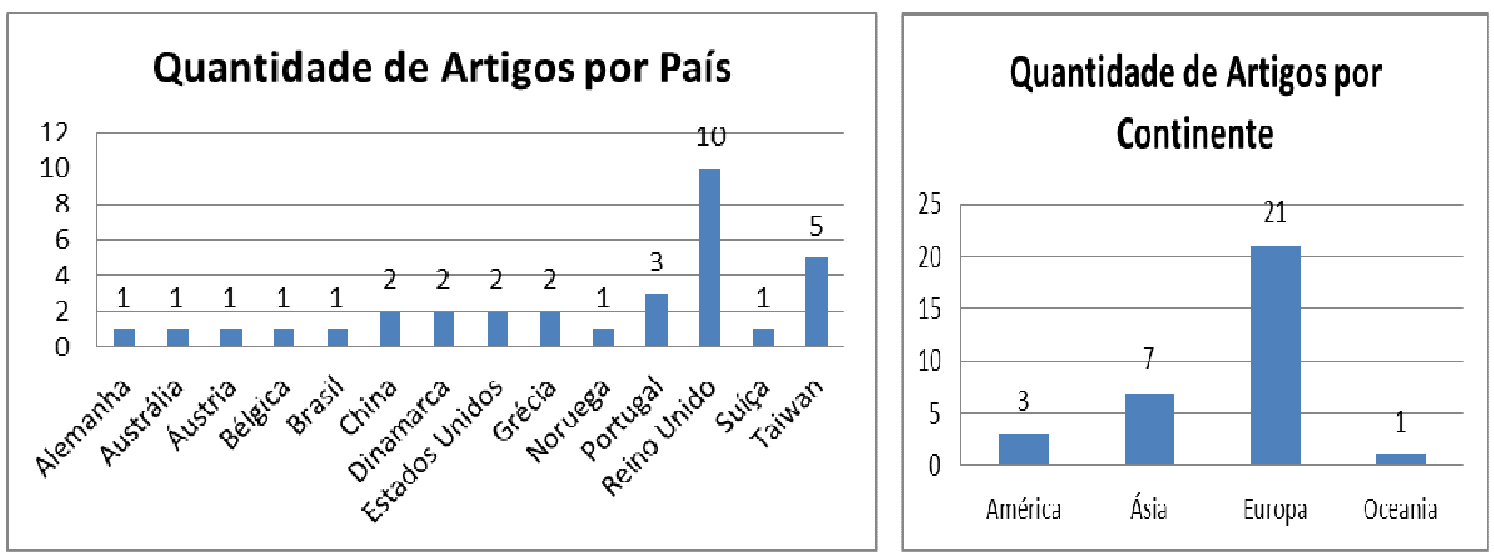

Figura 2. Quantidade de artigos pesquisados por país e continente

Para responder da P2 à P4, os critérios estabelecidos foram: apresenta método de desenvolvimento para jogos digitais de lógica ( $\mathrm{C} 1-\mathrm{S}$ se apresenta); apresenta algum método de desenvolvimento para jogos digitais (C2 - S se apresenta); apresenta jogos digitais de lógica desenvolvidos, porém não o método de desenvolvimento ( $\mathrm{C} 3-\mathrm{S}$ se apresenta); apresenta jogos digitais desenvolvidos, porém não o método de desenvolvimento (C4 - S se apresenta); apresenta um jogo digital de aprendizado (C5 $\mathrm{S}$ se apresenta); é relevante para os estudos desta pesquisa em níveis alto (apresenta o desenvolvimento de um método, inclusive de digitalização), médio (apresenta o estudo de um método para aprendizagem) e baixo (apresenta resultados da aplicação de um método), (C6 - A se alto, $\mathrm{M}$ se médio e B se baixo); e a abordagem - estudo de caso, revisão sistemática ou experimento $(\mathrm{C} 7$ - E se experimento, $\mathrm{C}$ se estudo de caso e $\mathrm{R}$ se revisão sistemática).

Os resultados da pesquisa a partir dos critérios estão apresentados no Quadro 2 (artigos A1 a A16) e no Quadro 3 (artigos de A17 a A31). Analisando-se os resultados, pode-se verificar que nenhum artigo aborda o $\mathrm{C} 1,16(52 \%)$ o $\mathrm{C} 2$, um (3\%) o C3, 14 (45\%) o C4 e 15 (48\%) o C5. Em relação à relevância deste estudo, 17 (55\%) possuem nível alto, nove (29\%) nível médio e cinco (16\%) nível baixo. Em relação à abordagem dos artigos estudados, $26(84 \%)$ são de experimento, seis (13\%) de revisão e um (3\%) de estudo de caso. 
Quadro 2. Resultado da pesquisa a partir dos critérios (A1 a A16)

\begin{tabular}{|l|c|c|c|c|c|c|c|c|c|c|c|c|c|c|c|c|}
\hline Sig & A1 & A2 & A3 & A4 & A5 & A6 & A7 & A8 & A9 & A10 & A11 & A12 & A13 & A14 & A15 & A16 \\
\hline C1 & & & & & & & & & & & & & & & & \\
\hline C2 & S & S & & S & S & S & S & & S & & & S & & & & \\
\hline C3 & & & & & & & & & & & & & & & S & \\
\hline C4 & & & S & & & & & S & & S & S & & S & S & & S \\
\hline C5 & S & S & S & & & & & S & & S & S & S & S & S & S & \\
\hline C6 & A & M & M & M & A & A & M & M & M & A & A & A & A & A & A & B \\
\hline C7 & E & E & R & R & E & E & C & E & E & E & E & E & R & E & E & R \\
\hline
\end{tabular}

Quadro 3. Resultado da pesquisa a partir dos critérios (A17 a A31)

\begin{tabular}{|l|l|l|l|l|l|l|l|l|l|l|l|l|l|l|l|}
\hline Sig & A17 & A18 & A19 & A20 & A21 & A22 & A23 & A24 & A25 & A26 & A27 & A28 & A29 & A30 & A31 \\
\hline C1 & & & & & & & & & & & & & & & \\
\hline C2 & S & S & S & & & & & & S & S & & & S & S & S \\
\hline C3 & & & & & & & & & & & & & & & \\
\hline C4 & & & & S & S & S & S & S & & & S & S & & & \\
\hline C5 & S & & S & S & & & & & & S & & & & S & \\
\hline C6 & A & A & A & A & B & B & B & M & A & A & B & M & A & A & M \\
\hline C7 & E & E & E & E & E & E & E & E & E & E & E & E & E & E & E \\
\hline
\end{tabular}

\section{Discussão}

Os resultados apresentados na Figura 1 e na Figura 2 sugerem que pesquisas que possuem alguma relação com métodos de desenvolvimento de jogos digitais de lógica intensificaram-se a partir de 2010, mais concentradas no continente Europeu, especialmente no Reino Unido, porém, em quantidade ainda mínima. Relacionando com os resultados do Quadro 2 e do Quadro 3, é possível verificar que dentre estas pesquisas, não há indícios de estudos dos métodos de desenvolvimento de jogos digitais de lógica.

Os resultados apresentados no Quadro 2 e no Quadro 3 sugerem que $48 \%$ dos estudos preocupam-se em realizar pesquisas para a apresentação de jogos digitais para o aprendizado, dentre os quais, $47 \%$ apresentaram uma metodologia de desenvolvimento desses jogos digitais voltados para o aprendizado. Os resultados mostram ainda que $52 \%$ dos estudos mencionam que as pesquisas preocupam-se com o estudo de métodos parciais ou totais quando se trata do desenvolvimento de jogos digitais.

Ainda com base nos resultados do Quadro 2 e do Quadro 3, 45\% das pesquisas envolvem o estudo de jogos digitais sem a apresentação da metodologia contra apenas $3 \%$ que envolvem o estudo de jogos digitais de lógica sem a apresentação de um método de desenvolvimento. Além disso, $52 \%$ dos estudos apresentam pesquisas com métodos que envolvem jogos digitais contra nenhuma pesquisa sobre estudos que envolvam os métodos de desenvolvimento de jogos digitais de lógica. 


\section{CBIE-LACLO 2015}

Anais do XXVI Simpósio Brasileiro de Informática na Educação (SBIE 2015)

Considerando apenas os artigos classificados no critério C6 como altamente relevantes para os estudos desta pesquisa e relacionados ao aprendizado, o A10 apresenta jogos digitais que verificaram a melhora do desempenho de resolução dos participantes a partir do uso de estratégias; o A11 apresenta a avaliação de um jogo educativo para a aprendizagem dos conceitos da tecnologia de informações e comunicações; o A14, A20 e A30 apresentam um modelo de jogo digital educacional e seu impacto no aprendizado; o A15 apresenta um jogo digital de lógica, os puzzles, e seu impacto no desenvolvimento de estratégias de resolução; o A25 apresenta uma abordagem que considera a narrativa nos jogos digitais; A29 apresenta um método de análise na criação de jogos digitais. Todos estes artigos podem agregar informações importantes para o desenvolvimento de um método para a transposição de jogos de lógica do meio físico para o digital.

Os artigos A5 e A19, que apresentam uma abordagem para design em animações 3D, A6, que apresenta novos métodos de design combinados aos elementos digitais baseados em jogos e A17 e A26, que apresentam um método de desenvolvimento de design para jogos digitais educacionais, podem contribuir com critérios de design no desenvolvimento de um método para jogos de lógica digitais. Em particular, o artigo A18, apresenta um modelo de desenvolvimento de jogos de tabuleiro digitais, não apenas com a preocupação do design, mas também da aprendizagem e das estratégias que os jogos de tabuleiro físicos possuem.

$\mathrm{O}$ artigo A1 apresenta um jogo educacional online promissor para a aprendizagem digital e o artigo A12 apresenta um método de desenvolvimento de um jogo digital em que os estudantes, além do aprendizado de conceitos matemáticos, desenvolvem a capacidade da criatividade e do raciocínio lógico. Ambos os artigos podem ser utilizados academicamente e cujo modelo de desenvolvimento do jogo pode contribuir para o desenvolvimento de métodos para jogos de lógica digitais.

O artigo A13 apresenta uma revisão dos jogos digitais voltados para educação. Neste artigo, foram encontrados apenas oito estudos relevantes para o aprendizado das ciências a partir dos jogos digitais, o que mostra que estudos sobre jogos digitais para o aprendizado, inclusive os de lógica, devem ser incentivados.

\section{Conclusão}

Considerando a geração atual dos estudantes, faz-se necessário o estudo e o desenvolvimento de metodologias que considerem o desenvolvimento de jogos digitais de lógica que possam ser utilizados pelos estudantes, de forma a garantir um aprendizado lógico, cognitivo, social e de planejamento.

A partir deste estudo de revisão, foi possível verificar que há estudos isolados de métodos de desenvolvimento de jogos digitais, porém, os estudos que utilizam ou definem os métodos para o desenvolvimento de jogos digitais de lógica, considerando o conjunto de todos os jogos de lógica, ainda não foram explorados pela literatura.

Os estudos que envolvem jogos digitais de aprendizagem têm crescido nos últimos anos, porém, os estudos que englobam os jogos digitais de lógica ainda precisam ser intensificados. Por este motivo, estudos de metodologias que tratam dos jogos digitais de lógica podem ser oportunidades de pesquisa. 
CBIE-LACLO 2015

Anais do XXVI Simpósio Brasileiro de Informática na Educação (SBIE 2015)

\section{Referências}

Biolchini, J., Mian, P. G., Natali, A. C., Travassos, G. H. (2005) "Systematic Review in Software Engineering: Relevance and Utility", Technical Report ES-679/05, PEC/COPPE/UFRJ.

Chen, H., Jian, C., Lin, W., Yang, P., Chang, H., (2014) "Design of Digital Game-Based Learning in Elementary School Mathematics," Ubi-Media Computing and Workshops (UMEDIA), 2014 7th International Conference on, pp. 322-325, 12-14

Cuperschmid, A. R. M.; Hildebrand, H. R. (2013) "Heurísticas de Jogabilidade Usabilidade e Entretenimento em Jogos Digitais". Marketing Aumentado. Campinas.

Dantas, V.; Nogueira, A.; Alisson, N.; Raniery, D.; Raul, J.; Soares, R.; Sampaio, R.; Kelson, W.; Costa T. (2013) Uma metodologia para estimular o raciocínio lógico baseada na reflexão crítica e no uso de jogos digitais. CBIE 2013 - II Congresso Brasileiro de Informática na Educação. Campinas.

Fraiman, L. (2013) "Como ensinar bem as crianças e adolescentes de hoje" 1a edição. São Paulo: Editora Esfera.

Fernandes, J. C. L. (2010) Educação Digital: "Utilização dos Jogos de Computador como Ferramenta de Auxílio à Aprendizagem". Fasci-Tech - Periódico Eletrônico da FATEC - São Caetano do Sul. V. 1. N. 3. p. 88-97. São Caetano do Sul.

Ramos, D. K. (2013) "Jogos Cognitivos Eletrônicos: Contribuições à Aprendizagem no Contexto Escolar". Ciência e Cognição. V. 18. N. 1. p. 19-32. Florianópolis.

Freire, P. (2011) "Ação cultural para a liberdade e outros escritos". 14a edição. Rio de Janeiro: Paz e Terra.

Hsu, Y., Shih, J. (2012) "Landscape Montage" Digital Game for Virtual Counseling Purposes," Digital Game and Intelligent Toy Enhanced Learning (DIGITEL), 2012 IEEE Fourth International Conference on, pp. 197-201, 27-30

Kitchenham, B. (2004) "Procedures for Performing Systematic Reviews". Keele, UK, Keele Universitu 33. p. 1-26.

Kitchenham, B., Brereton, O. P., Budgen, D., Turner, M., Bailey, J., Linkman, S. (2009) "Systematic Literature Review in Software Engineering - A Systematic Literature". Information and Software Technology 51 (1). p. 7-15.

Mäyrä, F., Holopainen, J., Jakobsson, M. (2012) "Research methodology in gaming: An overview". Simul. Gaming, 43(3), 295-299.

Mohebzada, J.G., Bhojani, A.H. (2011) "The cubes: A tangible game-based learning system," Innovations in Information Technology (IIT), 2011 International Conference on, pp.179-184, 25-27.

Ott, M., Pozzi, F. (2011) "Digital games as creativity enablers for children". Behaviour \& Information Technology, 1-9 (iFirst Article).

Sato, A. K. O. (2009) "Mapa do Jogo - Do Mundo Real ao Mundo Ficcional: A Imersão no Jogo". São Paulo: Cengage Learning. 